\title{
Optical and Structural Properties of CdS/PVA Nanocomposites
}

\author{
H.F. Al-Taay, Zainab J. Shanan,Eman khaddum, Nisreen Kh.abdalameer, \\ Sara Talal, Rajaa Nader \\ Department of Physics, College of Sciences for Women, University of Baghdad, Baghdad, Iraq.
}

\begin{abstract}
In this project, poly vinyl alcohol (PVA) polymer is used to prepare CdS/PVA nanocomposites via chemical deposition method. The samples were prepared using cadmium chloride $\mathrm{CdCl}_{2}$ as cadmium ions source while thiourea was used as a sulphur ions source and the yellow obtained precipitations too, which form PVA capped CdS nanoparticles. CdS/PVA nanocomposites samples were prepared by changing the molar concentration of thiourea of $(5,10,15$ mmolar). The surface morphology and crystallite sizes were determined through atomic force microscopy (AFM) measurements. The optical properties of the prepared samples were investigated by UV-Vis spectrophotometer and the photoluminescence $(P L)$ spectra. The structural properties of the obtained samples were characterized by X-ray diffraction (XRD) method.
\end{abstract}

Keywords: CdS, PVA, Chemical Path Deposition, XRD , AFM

\section{Introduction}

Nanomaterials have recently received much attention because of their unique physical and chemical properties. The control on the fundamental physical properties of materials can be achieved without altering their chemical composition by controlling the size, structure type, and surface states of the nanocrystalline materials [1,2]. Semiconductor nanoparticles-polymer composites have drawn tremendous attention due to their usage in various optoelectronic applications [3]. The synthesis and characterization of semiconductor nanoparticles have recently gained tremendous attention owing to their unique chemical and physical properties beside the huge potential for practical application of the composite system incorporating the nanoparticles. These properties differ significantly from those of the bulk material, which is related to the very small size of particle and coupled with high surface to volume effects $[1,4]$.

CdS/PVA nanocomposites (NCs) have attracted considerable attention due to wide direct band gap $(2.42 \mathrm{eV})$ of $\mathrm{CdS}$ along with good mechanical, acoustic and elastic properties of PVA. Polymer matrix provides stability, long shelf life and good control over the growth of nanoparticles. Optical properties of CdS/PVA are extensively studied due to their applications in wide technological fields [3,5,6]. Furthermore, nanocomposites have gained a great deal of interest since polymers as a host provides long term stability, flexibility and processability coupled with the functional tenability and control growth of nanoparticles; in addition, nanosized particles enhance their optoelectronic properties, allowing coupling between mechanical and optoelectronic properties [7, 8].

As a result, the polymer matrix has been frequently used to produce semiconductor-polymer nanocomposites. The polymer host matrix plays to confine the growth of nanoparticles and prevents aggregation. Many applications of nanoparticles need stable and rigid forms, such as thin films. The Usage of polymer as the matrix for nanoparticle additions enables easy fabrication of nanocomposites films [9].

The matrix material properties are significantly affected in the vicinity of the reinforcement note that with polymer nanocomposites, properties related to local chemistry, degree of thermoses cure, polymer chain mobility, polymer chain conformation, degree of polymer chain ordering or crystallinity can all vary significantly and continuously from the interface with the reinforcement into the bulk of the matrix[10]. The objective of the present work is to synthesize CdS/PVA nanocomposites via chemical deposition method, the effect of the molar concentration of thiourea on the morphology, crystalline structure, and optical properties of the prepared CdS/PVA nanocomposites is investigated.

\subsection{Preparation}

\section{Experimental Part}

CdS/PVA nanocomposites samples were synthesized as fallows first of all: a suitable quantity of PVA powder was dissolved in $100 \mathrm{ml}$ of distillate water and the resulting solution was gently mixed using magnetic stirrer for 1 hour. $0.05 \mathrm{M}$ aqueous solution of cadmium chloride $\left(\mathrm{CdCl}_{2}\right)$ and thiourea (TU) were used to prepared $\mathrm{CdS}$ as source of $\mathrm{Cd}$ and $\mathrm{S}$ respectively. Then, 5 mmolar solution of $\mathrm{CdCl}_{2}$ was added to PVA after that $\mathrm{NaOH}$ solution was added to the mixture raising the $\mathrm{pH}$ to 12 . As the final step, the thiourea was added in the final step to the mixture. Using buffer solution in the reaction is important to control the synthesis process. 
Three CdS /PVA nanocomposite samples were prepared by changing the concentration thiourea of $5,10,15$ mmolar).

\subsection{Characterization}

The structural properties of samples were investigated by XRD model (X'Pert Pro MPD by PANalytical Company, with $\mathrm{CuK} \alpha$ radiation). The XRD patterns of thin films and powder were recorded at room temperature. The optical properties were measured by using a photoluminescence (PL) spectra and UVVis Spectrophotometer model (Thermospectronic helios $\alpha$ V4.60) and PL spectra was investigated using PERKIN ELMER LS-3 fluorescence spectrometer and the samples are excited by $350 \mathrm{~nm}$ wavelength light.

\subsection{Surface Morphology}

\section{Results And Discussion}

Figure(1) shows the AFM images of prepared CdS/PVA nanocomposites with different molar concentration of thiouria. Analysis the AFM images showed that the surface roughness for synthesized sample decrease with molar concentration due to decreased particle size. Also, high density with good adherence to the substrate.

The diameter distribution for the prepared samples as shown in Figure (2) revealed that the average particle size decreased with increased the concentration ratio to 1:3 which may be due to the increased the film crystallizes. The average particle size was measured using AFM images that which (93nm) and (134nm)for CdS/PVA nanocomposites prepared using 1:1 Cd/S molar concentration and 1:3 Cd/S molar concentration respectively.
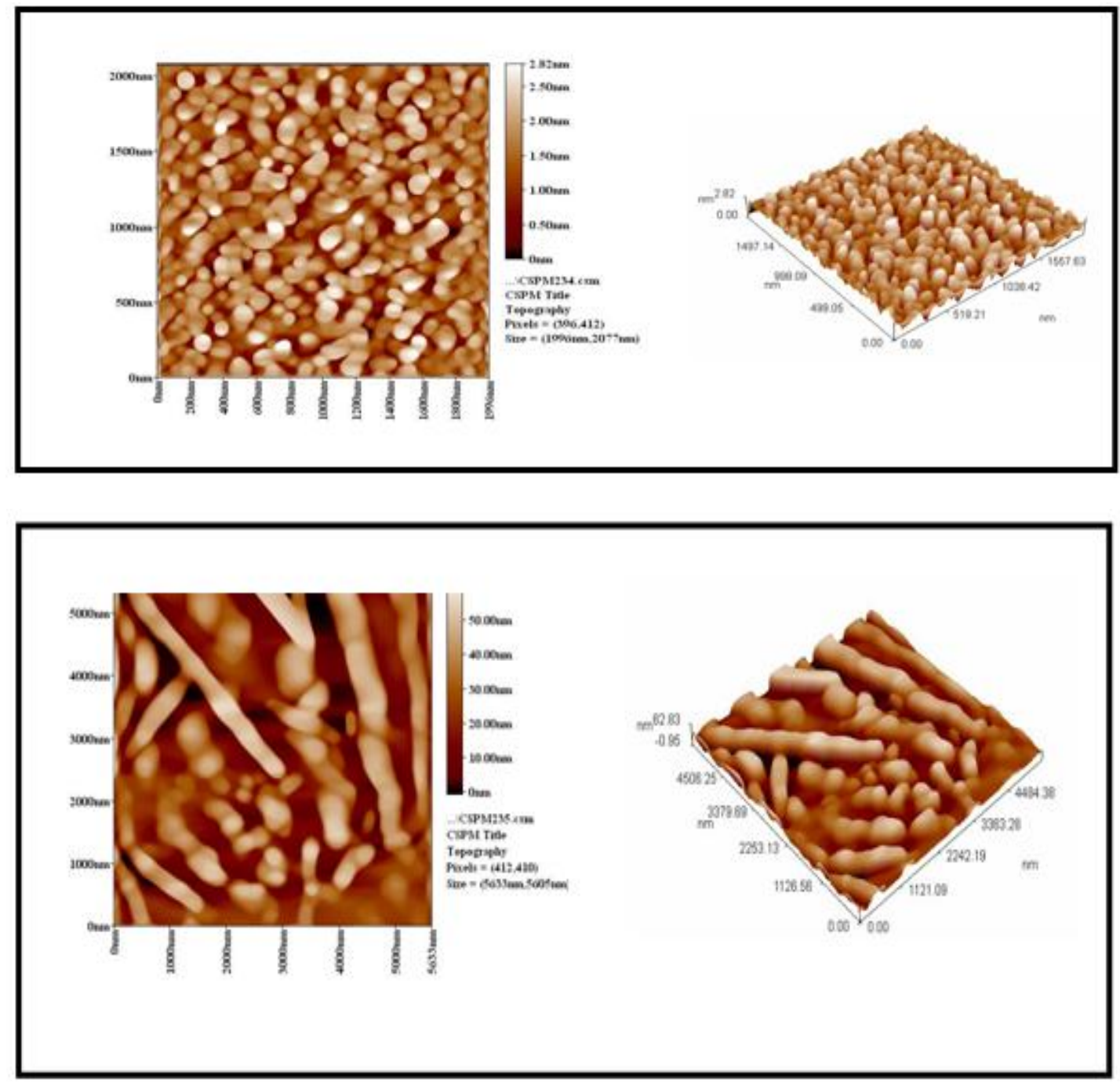

Fig. 1: AFM images of CdS/PVA nanocomposites prepared using (A) 1:1 Cd/S molar concentration, (B) 1:3 $\mathrm{Cd} / \mathrm{S}$ molar concentration 

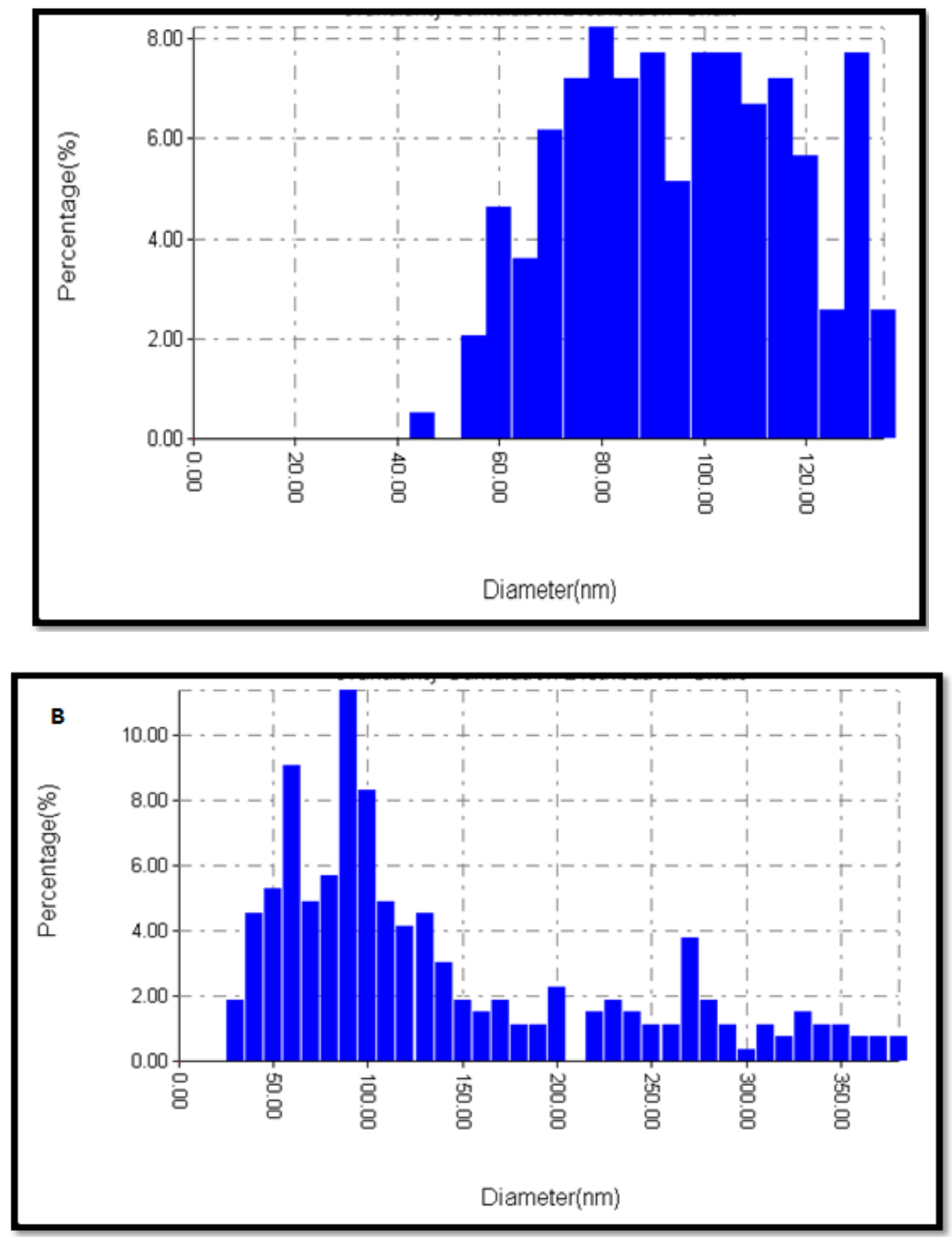

Fig. 2: Diameter distribution of CdS/PVA nanocomposites prepared using (A) 1:1 Cd/S molar concentration, (B) $1: 3 \mathrm{Cd} / \mathrm{S}$ molar concentration.

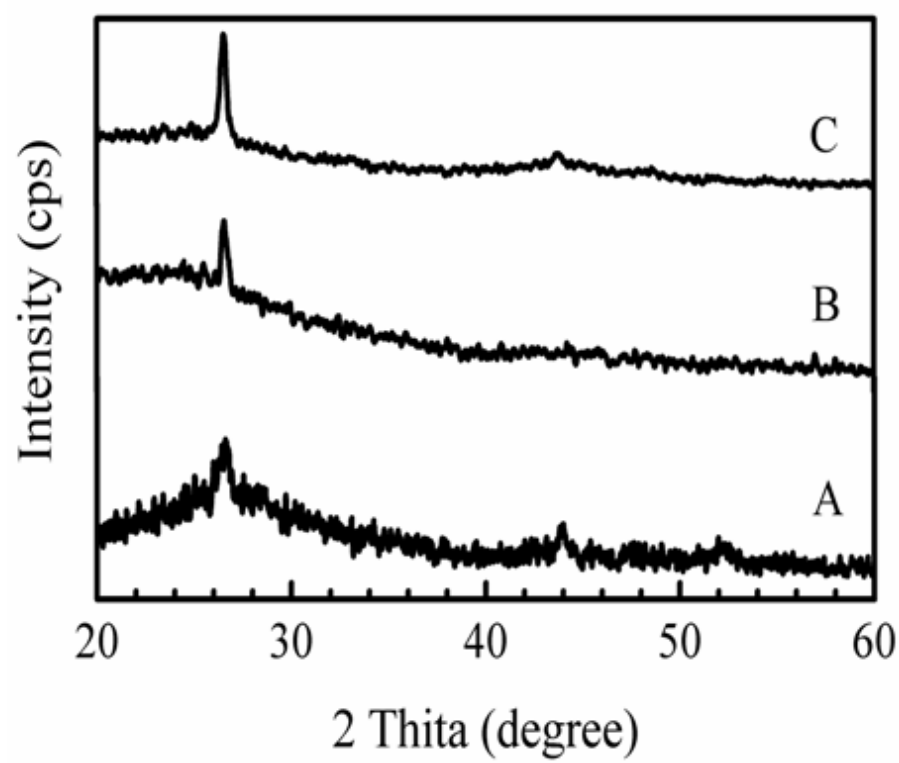

Fig. 3: XRD patterns of CdS/PVA nanocomposites prepared using (A) 1:1 Cd/S molar concentration, (B) 1:2 $\mathrm{Cd} / \mathrm{S}$ molar concentration, and (c) 1:3 Cd/S molar concentration 


\subsection{Structural properties}

Figure (3) shows XRD patterns of CdS/PVA nanocomposites prepared with different molar concentration of thiouria. The XRD shows diffraction pecks at $26.5^{\circ}$ and $44.0^{\circ}$ corresponding to (111) and (220) plans of zincblend (cubic) structure of CdS. In addition, the broad in diffraction peck for the ratio 1:1 indicates the smallest particles size which increased with increasing the concentration ratio to 1:3.

\subsection{Optical properties}

\subsubsection{Photoluminescence (PL) spectra}

The photoluminescence (PL) spectra of nanocrystalline materials is one of the most effective tools for diagnosing the structure quality, surface states, and impurity levels inside the optical band gap [11 ]. Figure (4) shows the PL spectra of the CdS prepared at various thiourea concentration. The CdS/PVA nanocomposites sample that prepared using low molar concentration of TU shows emission band peaked at 490nm while the samples that prepared using 1:2 and 1:3 of $\mathrm{Cd} / \mathrm{S}$ molar concentration emitted bands at $525 \mathrm{~nm}$ and $520 \mathrm{~nm}$, respectively. However, the blue shift in emission band for the sample that prepared using 1:1 ratio is due to the smallest particles size that led to increase in the optical bandgap.

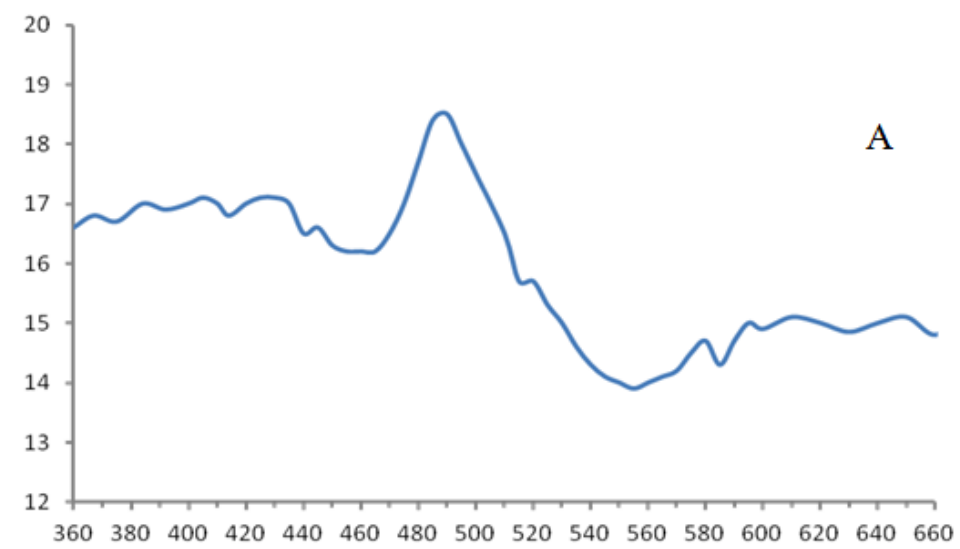

Wavelength (nm)

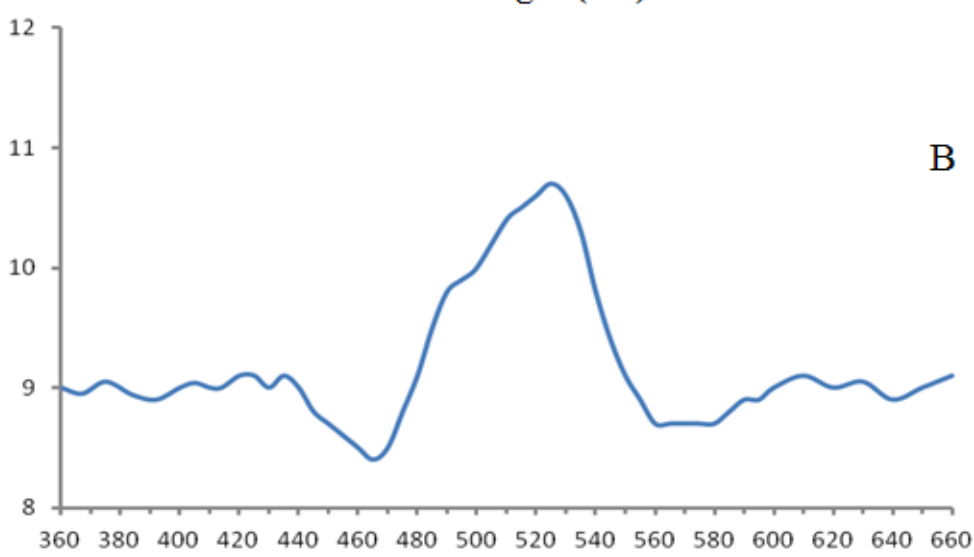

Wavelength (nm)

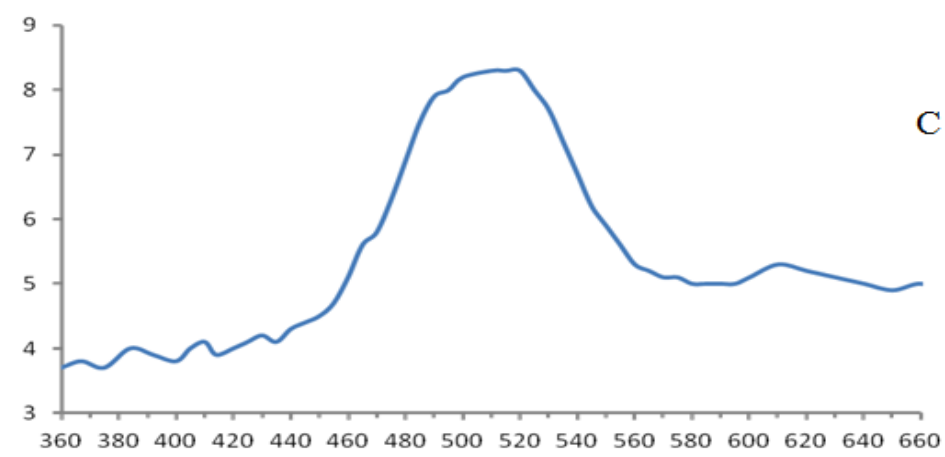




\subsubsection{UV-Vis Spectrophotometer}

The UV-vis absorption spectra of the CdS/PVA nanocomposites prepared at various molar concentration thiourea of $(5,10,15$ mmolar) with wavelengths in the range of 300-850nm are shown in Figure ( 5). It is evident from the spectra that, absorbance edge of all the prepared samples is depends on the molar concentration of thiourea relative to the bulk CdS optical band gap of $2.42 \mathrm{eV}(520 \mathrm{~nm})$. Absorbance coefficient $\alpha$ associated the strong absorption region of the film was calculated from absorbance (A) and the film thickness (t) using below relation [ 12$]$

$\alpha=2.3026 \mathrm{~A} / \mathrm{t}$.

The absorption coefficient $\alpha$ was analyzed using the following expression for optical absorption of semiconductors [12] $(\alpha h v)=\mathrm{A}(\mathrm{h} v-\mathrm{Eg})^{\mathrm{n} / 2}$

Where $\mathrm{A}$ is constant, Eg is separation between valence and conduction bands and $\mathrm{n}$ is constant that is equal to 1 for direct band gap semiconductor. The band gap values were determined from the intercept of the straight-line portion of the $(\alpha h v)^{2}$ against the $(h v)$ graph on the $h v$-axis using computer fitting program as shown in Figure (6) . The linear part shows that the mode of transition in these samples is the direct transition. The calculated band gap value of the sample prepared at various molar concentration of thiourea of $(5,10,15$ mmolar) were $(2.55,2.42,2.4 \mathrm{eV})$ respectively.

The band gap value decrease when the thiourea molar concentration increase as compare with the band gap for bulk CdS $(2.42 \mathrm{eV})$, the CdS sample prepare at 5 mmolar concentration has the higher value of bandgap which indicted the sample has the smallest particles size. According to effective mass model, the nanoparticles show of quantum confinement effect of the carriers [13]. From XRD patterns of the prepared samples, the intensity of the XRD increased as TU molar concentration increased which mean increasing in the particles size that in turn consisted with optical bandgap behavior.
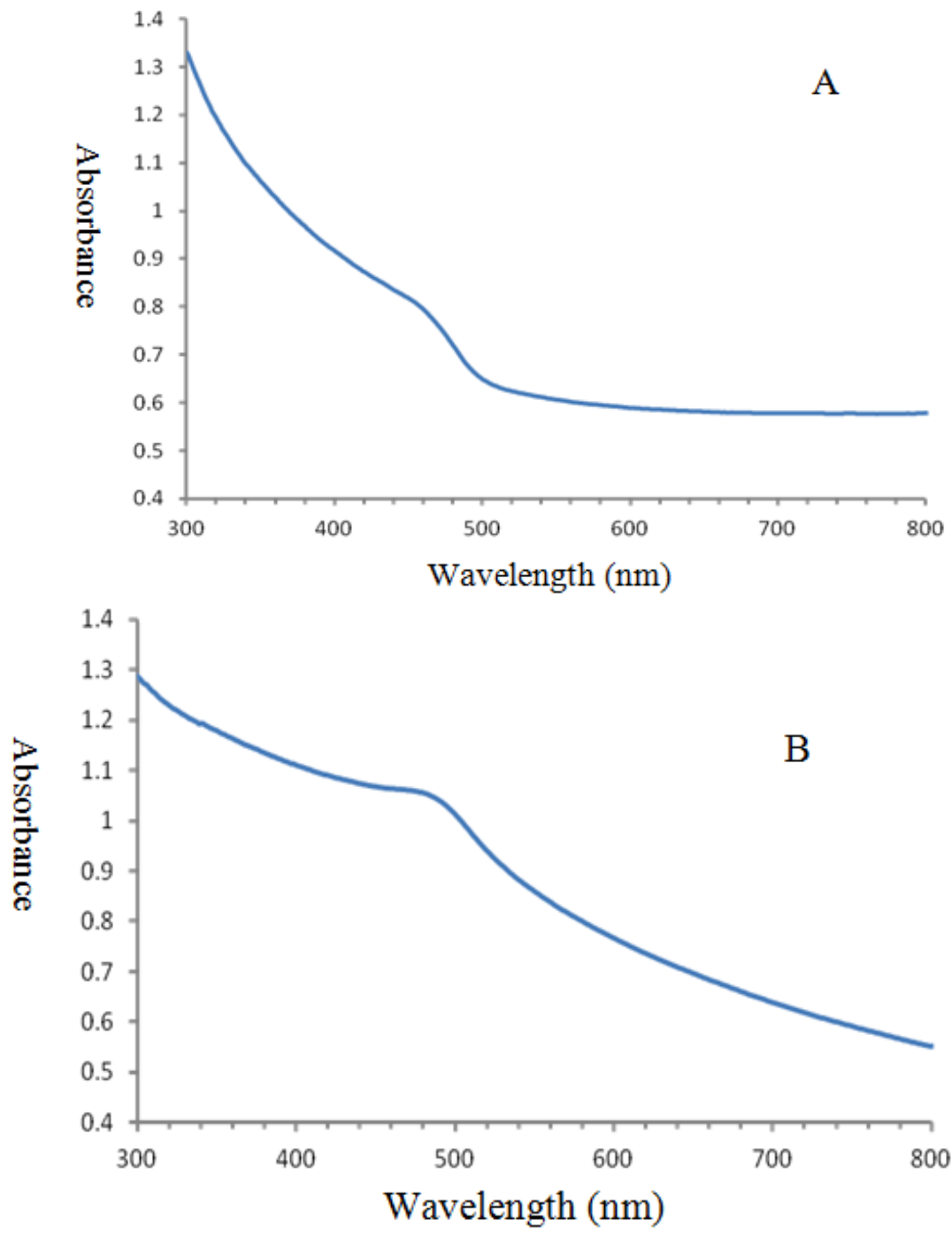


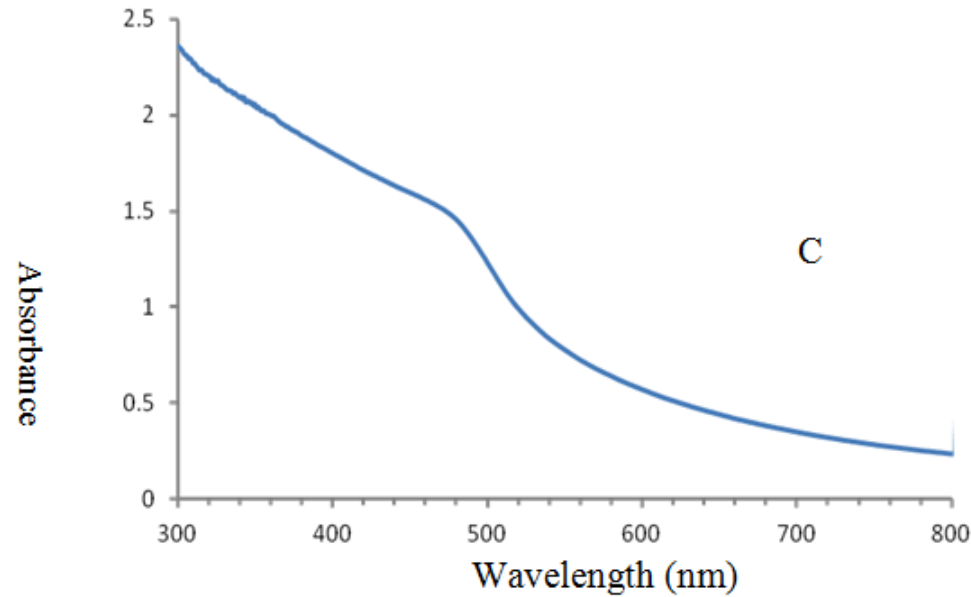

Fig 5: UV-Visible absorption of CdS/PVA nanocomposites prepared using (A) 1:1 Cd/S molar concentration,

(B) $1: 2 \mathrm{Cd} / \mathrm{S}$ molar concentration, and (c) $1: 3 \mathrm{Cd} / \mathrm{S}$ molar concentration
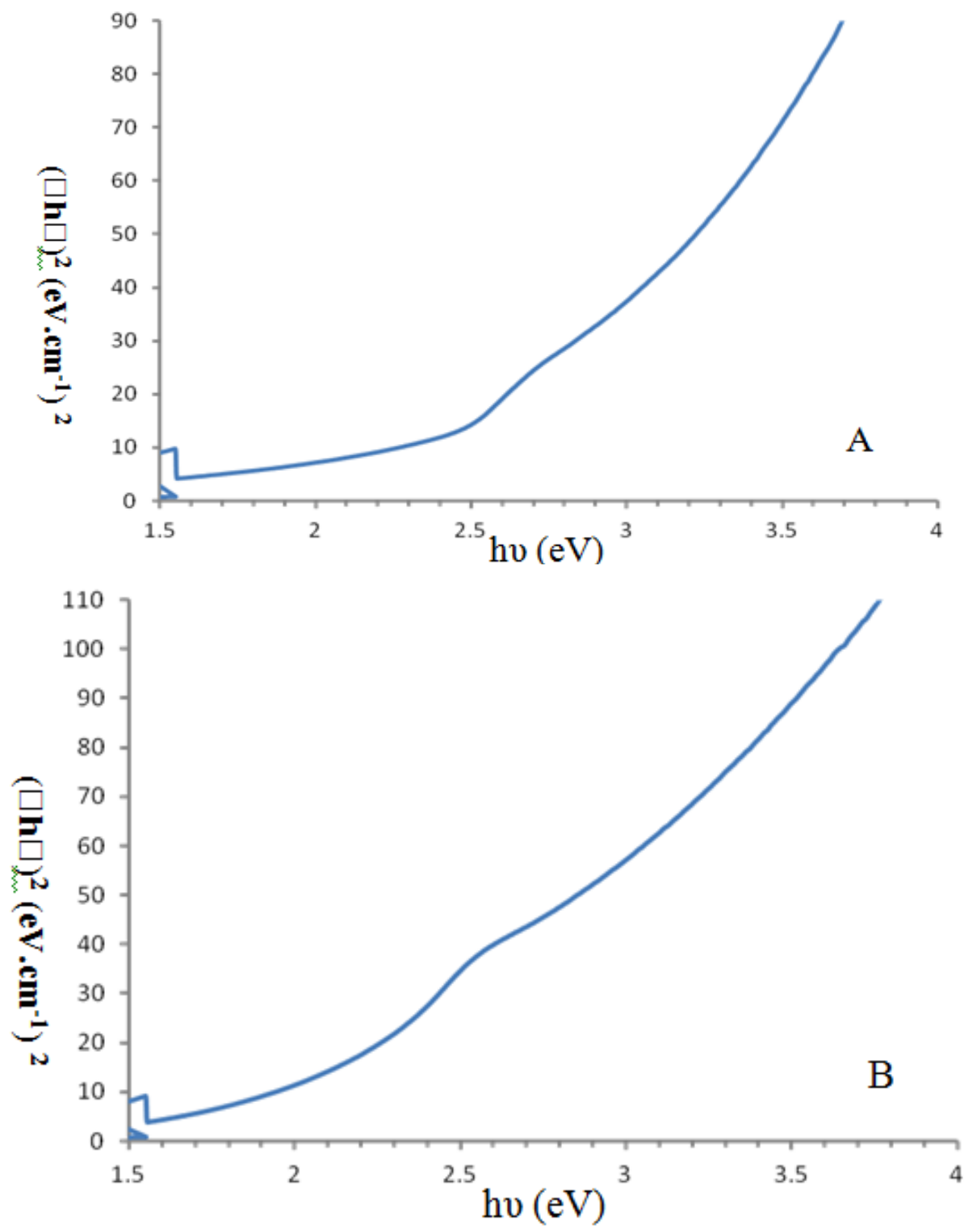


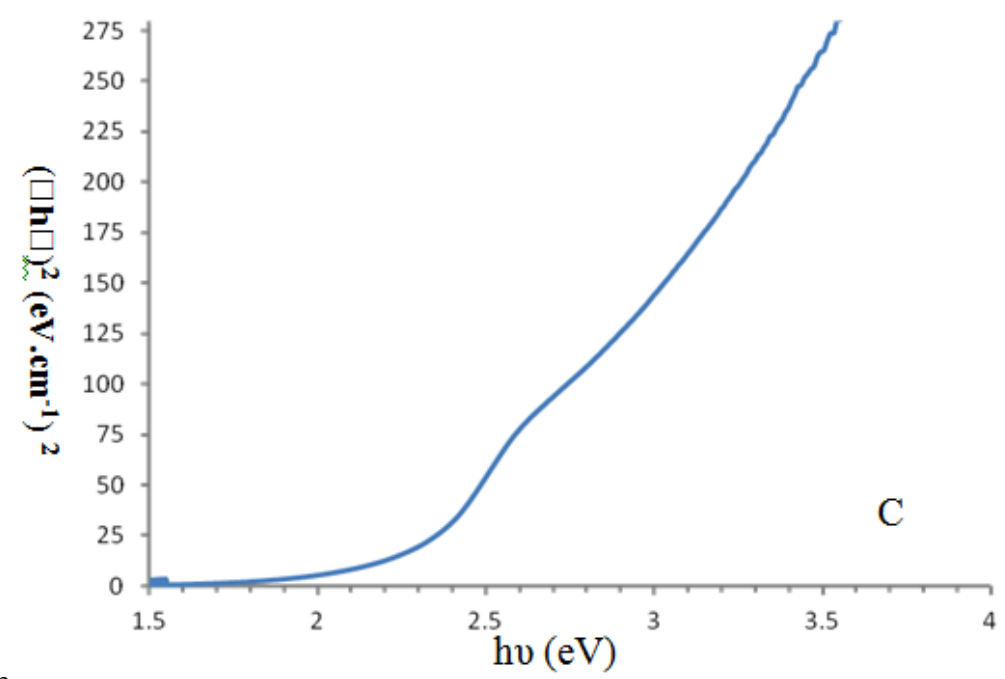

Fig 6: hv vs. $(\alpha . h v)^{2}$ of CdS/PVA nanocomposites prepared using (A) 1:1 Cd/S molar concentration, (B) 1:2 $\mathrm{Cd} / \mathrm{S}$ molar concentration, and (c) $1: 3 \mathrm{Cd} / \mathrm{S}$ molar concentration

\section{Conclusions}

CdS/PVA nanocomposites samples were synthesized using chemical deposition method using various thiourea concentration of $(5,10,15$ mmolar). The AFM images showed that the surface roughness for synthesized sample decrease with molar concentration with average particle size of $(93 \mathrm{~nm})$ and $(134 \mathrm{~nm})$ for CdS/PVA nanocomposites prepared using 1:1 Cd/S molar concentration and 1:3 $\mathrm{Cd} / \mathrm{S}$ molar concentration respectively.

XRD patterns show that all of prepared samples are formed as zincblend (cubic) structure. The diffraction peak which corresponds to (111) plane be sharper when the molar concentration of thiourea was increased indicating to enhance the crystallinity and increasing the particles size of CdS. PL spectra CdS/PVA nanocomposites that synthesis using concentration ratio 1:1 shows blue shift in emission band comparing by the other samples. However, the blue shift in emission band is due to the small particles size that led to increase the optical bandgap as its confirms by UV-Vis measurements. The value of band gap decrease from $2.55 \mathrm{eV}$ to $2.4 \mathrm{eV}$ when the molar concentration of thiourea increases from 1 to 3 mmolar.

\section{Referances}

[1] Koo, H.J. (2006) Polymer nanocomposites processing characterization and applications. USA: In: McGraw Hill.

[2] H.F. Al-Taay, M.A. Mahdi, D. Parlevliet, Z. Hassan, P. Jennings, Superlattices and Microstructures, 61 (2013) $134-145$.

[3] V. Bala, S.K. Tripathi, R. Kumar, Materials Letters, 132(2014)38-40

[4] H. Zhao, E. P. Douglas, B. S. Harrison, K. S. Schance. Preparation of CdS Nanoparticles in Salt-Induced Block Copolymer Micelles. Langmuir 2001; 17: 8428-8433.

[5] R.Maity, , K.K. Chattopadhyay.Synthesis and optical characterization of CdS nanowires by chemical process. J. Nanopart. Res. 2006; 8: 125-130

[6] W. Qingqing, X. Gang and H. Gaorong. Solvothermal synthesis and characterization of uniform CdS nanowires in high yield. Jr of Sold. St. Chem. 2005; 178(9): 2680-2685.

[7] J. D. Patela and T. K. Chaudhuri. Synthesis of PbS/poly (vinyl-pyrrolidone) nanocomposite. Materials Research Bulletin 2009; 44(8):1647-1651.

[8] 8-H. Wang, P. Fang, Z. Chen, S. Wang, Applied Surface Science, 253, (2007) 8495-8499.

[9] A, Kharazmi. E, Saion. N, Faraji. R, M, Hussin. W,M,M,Yunus. Radiation Physics and Chemistry,97(2014)212-216

[10] M. Pattabi, B. S. Amma and K. Manzoor. hotoluminescence study of PVP capped CdS nanoparticles embedded in PVA matrix. Materials Research Bulletin 2007; 42: 828-835

[11] M.A. Mahdi, J.J. Hassan, S.S. Ng, Z. Hassan, N.M. Ahmed, Physica E 44 (2012) 1716-1721.

[12] Longhurst, R.S. 1957. Geometrical and Physical Optics, Longmans Green, London

[13] M.A. Mahdi, Z. Hassan, S.S. Ng,J.J. Hassan, S.K. Mohd Bakhori, Thin Solid Films 520(2012)3477-3484 